\title{
Estado de conservación de las especies forestales amenazadas, abarco, jigua negro, guayaquil, guayacán amarillo y pino amarillo en los municipios chocoanos de Riosucio, Carmen del Darién, Istmina, Río Quito y Juradó
}

\section{State of conservation of the threatened forest species, abarco, jigua negro, guayaquil, guayacán amarillo y pino amarillo in the chocoanos municipalities of Riosucio, Carmen of the Darién, Istmina, Río Quito and Juradó}

\section{William Klinger Brahan ${ }^{*}$}

\section{RESUMEN}

Con el propósito de levantar información que diera cuenta del estado de conservación de las especies forestales amenazadas, abarco (Cariniana pyriformis), jiguanegro(Ocotea cernua), guayaquil(Centrolobiumparaense), guayacán amarillo (Tabebuia crysantha) y pino amarillo (Podocarpussp.)en cinco municipios del departamento del Chocó, Riosucio, Carmen del Darién, Istmina, Río Quito y Juradó, el Instituto de Investigaciones Ambientales del Pacífico y la Corporación Autónoma para el Desarrollo Sostenible del Chocó, constituyeron una alianza de cooperación horizontal que posibilitó la ejecución del proyecto; se buscaba tener bases sólidas para la toma de decisiones relacionadas con el uso y manejo de estas especies. El proyecto recibió apoyo financiero del Fondo de Compensación Ambiental de Colombia. Durante la fase de campo se utilizó un muestreo diagnóstico, se establecieron 1349 parcelas de 10m $\times 10$ m equivalentes a 13,49 has, distribuidas por zona de la siguiente manera; 205 en Istmina, 660 en Juradó, 226 en Riosucio, 62 en Carmen del Darién y 196 en Río Quito. De conformidad con los resultados obtenidos en la superficie muestreada las especies jigua negro y abarco mostraron mayor representatividad, el guayacán amarillo tuvo un representatividad media, y al contrario de estas las especies, el guayaquil y el pino amarillo registraron una significativa menor presencia, denotando así su condición de especies realmente amenazadas. Con fundamento en los resultados se proponen tres categorías de manejo para las especies forestales amenazadas, recuperación con veda de largo plazo, preservación con veda de mediano plazo y conservación en el corto plazo; estas orientaciones de manejo se deben aplicar conforme a las condiciones de cada especie en cada sitio. Se recomienda avanzar de manera inmediata en el establecimiento de las vedas, al tiempo que se incorporan otros municipios del departamento a estudios de esta naturaleza.

Palabras clave: Especies forestales amenazadas; Abarco; Pino amarillo; Jigua negro; Guayacán amarillo; Guayaquil.

\begin{abstract}
With the purpose of raising information that would track the state of conservation of threatenedforest species, covers (Cariniana pyriformis), black Jigua (Ocotea cernua), Guayaquil (Centrolobium para), guayacán yellow (Tabebuia crysantha) and yellow pine (Podocarpus sp.) in five municipalities in the
\end{abstract}

* Ingeniero Forestal, MSc. Profesor Titular de la Universidad Distrital Francisco José de Caldas, Facultad de Medio Ambiente y Recursos Naturales, Grupo de Investigación PROPOBOS y Director del Instituto de Investigaciones Ambientales del Pacífico, Quibdó, Colombia.e-mail:wklinger@distrital.edu.co

Recibido: marzo 12, 2009 Aceptado: marzo 26, 2009 
department of Chocó, Riosucio, Carmen del Darién, Istmina, Rio Quito and jury, the IIAP and CODECHOCO, formed an alliance of horizontal cooperation that enabled the implementation of the project. During the field sampling was used diagnostic plots were established $134910 \mathrm{~m} \times 10 \mathrm{~m}$ equivalent to 13.49 hectares, distributed by zone as follows; Istmina 205, 660 Juradó, 226 in Riosucio, 62 in Carmen del Darien and 196 in Río Quito. In accordance with the results obtained in the sampled surface jigua species showed black and covers more representative, the representative had a yellow guayacán media, and unlike these species, and yellow pine Guayaquil reported significantly less presence, and denoting their status as truly threatened species. Based on the results suggests three categories offorest management for threatened species, recovery from long-term ban, ban preservation in the medium term and short-term storage, these management guidelines should be applied according to the conditions of each species in each site.

Keywords: Endangeredforest species; Covers; Yellow pine; Black jigua; Guayacán yellow; Guayaquil.

\section{INTRODUCCIÓN}

La estratégica ubicación geográfica del departamento del Chocó y sus condiciones especiales de suelo y clima lo hacen albergue de importantes ecosistemas, que se conforman de especies de alto valor ecológico y comercial, en la mayoría de los casos asociado con las posibilidades de uso de la madera, dadas sus propiedades físicas, mecánicas, de durabilidad natural, de secado, de tratabilidad y de trabajabilidad. Las poblaciones de especies con un alto valor de uso local, regional, nacional y/o internacional van sufriendo una fuerte presión antrópica que las pone en peligro de agotamiento o de extinción, máxime cuando se suman a esa presión otras actividades productivas, que en algunos casos procuran la ampliación de la frontera agropecuaria, pero en otros, derrumban la selva para la extracción de otros recursos provenientes del suelo.

Tal es el caso de especies como el abarco (Cariniana pyriformis), jigua negro (Ocotea cernua) y pino amarillo (Podocarpus sp.) que por la calidad de sus maderas han sido sometidas a una intensa actividad de aprovechamiento en diferentes rincones del mundo, al punto que hoy se encuentran en libros rojos y en listados de la Unión Internacional para la Conservación de la Naturaleza (UICN). Como estrategia de uso adecuado y sostenible de las poblaciones de este tipo de especies, se requiere el levantamiento de información que permita conocer su estado de conservación, la ubicación espacial precisa de sus individuos, la situación fenológica de cada uno de ellos y su condición de madurez y desarrollo, en la perspectiva de establecer cuáles individuos son especial- mente útiles para futuros planes de repoblamiento, y además, cuáles áreas o espacios geográficos son los que con mayor urgencia necesitan ser intervenidos. Los municipios de Istmina, Riosucio, Carmen del Darién, Juradó y Río Quito, por su alta vocación forestal por la presencia de un gran número de especies maderables, han sido sometidos a intensos procesos de aprovechamiento forestal, de allí la importancia de escogerlos como sitios objeto de este estudio. Adicionalmente en los municipios de Istmina y Río Quito se desarrollan actualmente actividades de minería mecanizada que hacen mucho más complejo este panorama.

Las razones anteriores condujeron a establecer una alianza de cooperación horizontal entre el Instituto de Investigaciones Ambientales del Pacífico (IIAP) y la Corporación Autónoma para el Desarrollo Sostenible del Chocó (CODECHOCO), la primera como entidad cuyo objeto misional es la investigación y la segunda como institución encargada del ejercicio de la autoridad ambiental en el departamento del Chocó. El proceso de investigación contó con el apoyo económico del Fondo de Compensación Ambiental de Colombia y arrojó información de gran interés y utilidad en relación con el uso y manejo de las especies estudiadas.

\section{OBJETIVOS}

El proyecto de investigación se hizo con el propósito de levantar una línea base de información sobre las especies forestales nativas amenazadas, abarco, jigua negro y pino amarillo, que posibilitara la recomendación de medidas de manejo orientadas a la protección de estas especies en los municipios chocoanos de Istmina, Riosucio, Carmen del Darién, Río Quito y Juradó, tomando en cuenta específicamente los siguientes objetivos:

1. Registrar aspectos cualitativos en relación con la presencia de individuos y su estado de madurez, de las especies forestales nativas amenazadas, abarco, jigua negro y pino amarillo en los municipios de Río Quito: San Isidro; Istmina: Suruco y Puerto Salazar; Juradó: Resguardo Indígena de Juradó; Riosucio: Truandó Medio; y Carmen del Darién: Chicao.

2. Levantar y analizar información cuantitativa en cuanto a altura, diámetro y estado fenológico de las especies forestales nativas amenazadas, abarco, jigua negro y pino amarillo en los municipios de Río Quito: San Isidro; Istmina: Suruco y Puerto Salazar; Juradó: Resguardo Indígena de Juradó; Riosucio: Truandó Medio; y Carmen del Darién: Chicao.

\section{ASPECTOS METODOLÓGICOS}

El desarrollo del proyecto comprendió varias etapas íntimamente ligadas una a otra; se inició con un proceso de 


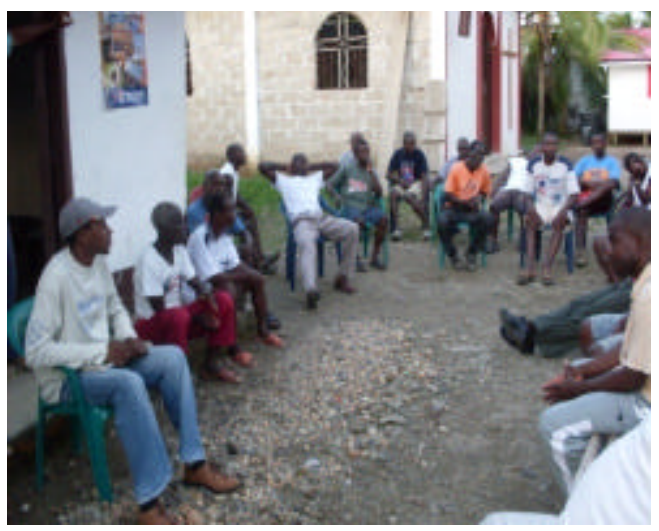

Figura 1. Aspectos de la concertación con la comunidad en los diferentes municipios

concertación, al tiempo que se emprendía una ardua revisión de información de la fase de campo; se levantó la información primaria con un trabajo que vinculó a miembros de la comunidad y finalmente se hizo el procesamiento de la información y la elaboración del documento final.

\section{CONCERTACIÓN}

Se realizó esta etapa con varios propósitos, en primer lugar, informar a los habitantes de cada área de estudio sobre las pretensiones del proyecto y sus resultados esperados, la metodología propuesta para desarrollarlo y los impactos que eventualmente se generarían con la aplicación de la información aportada y, en segundo lugar, lograr acuerdos en cuanto a los mecanismos de participación de la comunidad, definir los sitios precisos de muestreo y las rutas y costos necesarios para su acceso, y avanzar en la identificación del personal de la zona que podría vincularse al proyecto.

Para tal efecto se canalizaron los esfuerzos a través de los representantes legales de los consejos comunitarios mayores para luego llegar hasta los consejos comunitarios locales en el caso de comunidades negras y a través de la Asociación de Cabildos Indígenas Embera, Wounaan, Katio, Chami y Tule del Departamento del Chocó OREWA, para establecer el contacto con el Cabildo Indígena de Juradó, en el caso de las comunidades indígenas. Una vezidentificadas las comunidades en cuyo territorio se adelantaría el proyecto, se realizó una convocatoria amplia de los miembros de consejos y cabildos para llevar a cabo los talleres de concertación; en ellos se lograron los acuerdos previstos y se dispuso de los mecanismos para enfrentar la fase de campo del proyecto.

En la Figura 1 se pueden apreciar algunas evidencias del proceso de concertación, muestra registros fotográficos de las reuniones celebradas en cada una de las zonas de estudio, en la que se destaca la participación de los miembros de juntas directivas de los consejos comunitarios involucrados.

\section{PRODUCCIÓN DE CARTOGRAFÍA BÁSICA Y TEMÁTICA}

Para la producción de la cartografía básica, se recurrió a trabajos que con anterioridad había desarrollado el IIAP, en especial el proyecto de formulación de los Esquemas de Ordenamiento Territorial de los Municipios rezagados del Pacífico colombiano y el Proyecto Manglares, donde se digitalizó a escala 1: 25.000, la cartografía producida por el IGAC.

Tomando este referente, se elaboró un mapa de cada uno de los municipios objeto del proyecto, donde se identificó con un punto la zona de muestreo; luego se realizó un mapa ampliado de esta zona para lograr mayor detalle; en ellos se pueden identificar accidentes naturales hasta donde la escala de presentación lo permite y ubicación de los centros poblados de mayor importancia. Es precisamente sobre estos últimos mapas en donde se muestra la distribución espacial de las especies forestales identificadas en la etapa de campo, las cuales fueron georreferenciadas utilizando los GPS; además, una vez producidos los mapas temáticos con la distribución espacial de las diferentes especies, a cada mapa se le anexa una matriz, que indica la especie forestal, las coordenadas geográficas y planas del respectivo árbol al igual que su estado de desarrollo (fustal, latizal o brinzal).

El proceso metodológico que se siguió para la elaboración de cada mapa temático después de obtener la matriz con el nombre de la especie, coordenadas de cada árbol y grado de desarrollo fue el siguiente:

a. Ordenar la matriz de especies en orden alfabético.

b. Asignar un número en las columnas de grado de desarrollo (fustal, brinzal o latizal).

c. Filtrar las especies por grado de desarrollo.

d. Exportar las matrices mediante la utilización del software ArcGis en Dbase IV a la base cartográfica obtenida con anterioridad.

e. Asignar un ícono a cada especie para su identificación en el respectivo mapa temático.

f. Editar e imprimir.

g. Exportar los mapas temáticos en formato .jpg para ser insertados en los textos.

\section{LEVANTAMIENTO DE INFORMACIÓN SECUNDARIA Y PRIMARIA}

Se conformaron cuatro grupos de trabajo y en reunión de concertación del equipo general del proyecto, se le asignó a tres grupos de trabajo una zona de estudio y a un grupo de trabajo dos zonas de estudio, encomendándole el levantamiento de las características generales de cada zona, en cuanto a aspectos biofísicos y socioeconómicos en el entendido que esta información sería útil al momento de formular los 


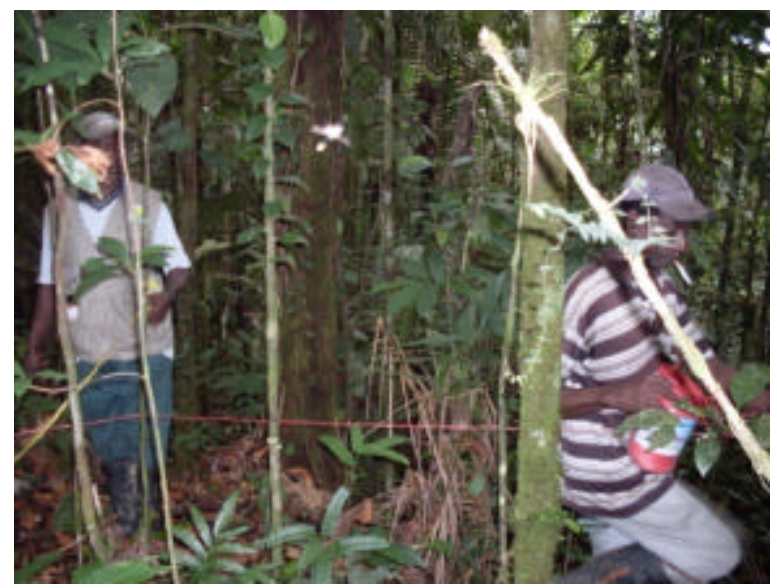

Figura 2. Detalles de la delimitación de las parcelas de muestreo

planes de manejo de las especies forestales nativas amenazadas; de igual manera se asignó la tarea de revisión de literatura sobre las especies forestales amenazadas. Los grupos de trabajo auscultaron en bibliotecas, bases de datos e internet hasta ubicar la información.

Una vez en campo, se seleccionaron equipos de apoyo local, procediendo a realizar charlas de intercambio que proporcionarán insumos básicos para un buen desarrollo de las actividades propuestas; en ese sentido el personal de apoyo ubicó los sitios de mayor ocurrencia de las especies forestales en estudio, seguidamente se realizó capacitación en algunos aspectos que se consideraron relevantes, adelantando prácticas de campo para consolidar el aprendizaje de los temas tratados (Figura 2). Los aspectos que se tuvieron en cuenta en el proceso de capacitación fueron:

Diligenciamiento de los formularios de campo.

Uso de los instrumentos de medición e interpretación del muestreo a utilizar.

Conocimiento de las funciones de cada uno de los integrantes del grupo.

Unificación de los criterios para la identificación de las especies forestales en particular la regeneración natural.

Definición de los criterios para la definición de la altura comercial y la calidad de fuste.

Definición de las categorías a inventariar, con respecto a los diámetros.

El método utilizado para la toma de información primaria fue el de muestreo diagnóstico, que permitió ajustarse a las condiciones específicas del trabajo; para el análisis estructural y florístico se establecieron 1.349 parcelas de $10 \mathrm{~m}$ x $10 \mathrm{~m}$ según la superficie total de cada zona, para un total de 13,49 h, 205 en Istmina, 660 en Juradó, 62 en Carmen del Darién, 226 en Río Sucio y 196 en Río Quito, en las cuales, una vez se identificó por parte de los reconocedores alguna de las especies en estudio, se procedió a delimitar las parcelas con

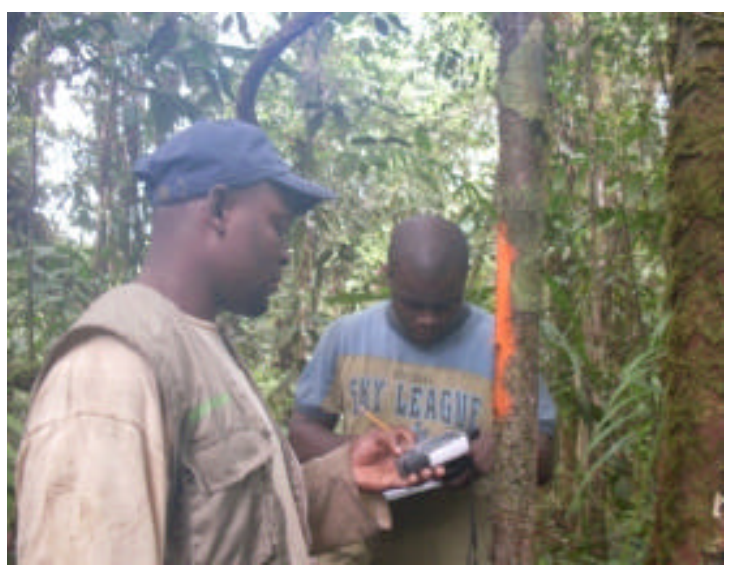

Figura 3. Georreferenciación de individuos de las especies amenazadas

cabuya, tal como se aprecia en la Figura 2, luego, en cada parcela, se hizo el levantamiento de la información correspondiente a los estados de brinzal, latizal y fustal.

Además del registro de la información correspondiente a las características de las especies forestales nativas amenazadas, con ayuda del equipo de georreferenciación satelital (GPS) se tomaron las coordenadas geográficas de ubicación de cada uno de los individuos inventariados y se procedió a marcarlos con pintura de aceite con el objeto de encontrarlos con mayor facilidad. La Figura 3 muestra evidencias de la georreferenciación de los individuos de algunas de las especies forestales amenazadas en cada uno de los cinco municipios del departamento del Chocó que se tuvieron en cuenta para el desarrollo del estudio.

Todos los individuos fueron registrados en un formato prediseñado que incluyó información sobre diámetro, altura comercial y altura total; estas corresponden a variables de tipo cuantitativo, de igual manera se registró información sobre la forma y tamaño de las copas, presencia de bejucos y estado fitosanitario, variables de tipo cualitativo que presentan relevancia en los diferentes estados del bosque. La Figura 4 muestra detalles del registro de la información en campo.

De igual manera se consideró pertinente la colección de material vegetal para la identificación de las especies, éstas fueron entregadas al herbario de la Universidad Tecnológica del Chocó, estamento que realizó la identificación hasta los niveles que se encuentran reportados en este documento. La Figura 5 muestra los detalles de la recolección y preparación de muestras botánicas. La compilación y tabulación de los datos registrado se realizó mediante la utilización de base de datos en Office Excel tomando como referente los cuadros y fórmulas establecidas en la guía técnica del MinAmbiente (2002), las cuales permitieron generar tablas y gráficas que facilitaron el análisis de la información. 


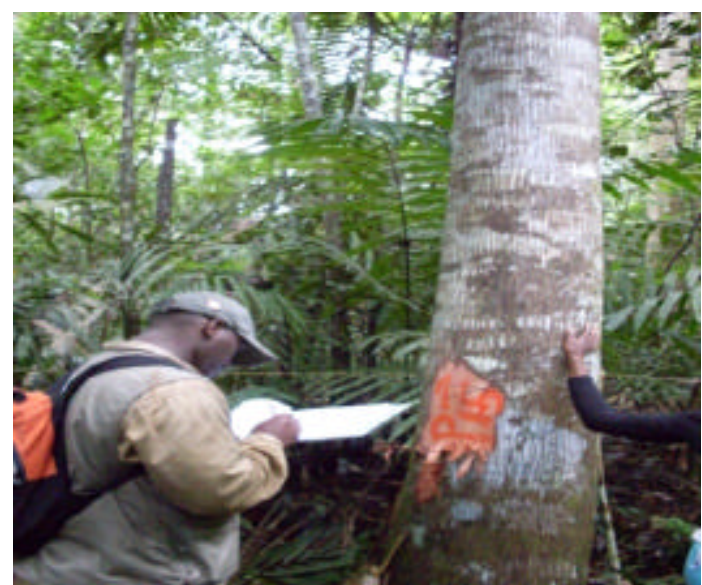

Figura 4. Registro de la información en campo

\section{PROCESAMIENTO DE LA INFORMACIÓN PRIMARIA}

El análisis estructural de la vegetación correspondiente a especies forestales amenazadas se realizó exclusivamente tomando en cuenta este tipo de especies en cada sitio, por lo que sus resultados no deben mirarse de manera absoluta, simplemente constituyen una manera de comparar la situación que en materia de escasez se encuentra en cada caso.

Las variables se calcularon así:

Densidad $=\mathrm{N}^{\circ}$ de individuos/área total del muestreo en hectáreas.

Abundancia relativa $=\left(\mathrm{N}^{\circ}\right.$ de individuos $/ \mathrm{N}^{\circ}$ de individuos en el área muestreada) $* 100$

Frecuencia $=\left(\mathrm{N}^{\circ}\right.$ de unidades de muestreo $/ \mathrm{N}^{\circ}$ total de unidades de muestreo) $* 100$

Frecuencia relativa $=($ Frecuencia absoluta/Sumatoria de las frecuencias absolutas) $* 100$

Dominancia relativa $=($ Área basal de cada especie/ Área basal total $) * 100$

Índice de valor de importancia IVI = Abundancia relativa $\%+$ Frecuencia relativa $\%+$ Dominancia relativa $\%$

\section{PRESENCIA DE LAS ESPECIES FORESTALES NATIVAS AMEZANADAS EN LOS MUNICIPIOS}

En la Tabla 1 se muestra marcada con X la presencia de las especies forestales nativas amenazadas abarco, jigua negro, guayaquil, guayacán amarillo y pino amarillo en las cinco zonas de trabajo, como preámbulo a la relación de información cualitativa por sitio de trabajo.

Independientemente de las cantidades en que se encuentren cada una de estas especies forestales amenazadas, el municipio de Juradó cuenta con el 100\% de ellas, razón de sobra para tener como zona estratégica a esta localidad, en especial los sitios donde se realizaron los muestreos, Resguardo Indígena Mayor de Juradó y Resguardo Indígena de Nussipurrú al momento de abordar programas de recuperación de este recurso biológico. De igual manera, el municipio de Istmina presenta el menor porcentaje en términos de la presencia de las cinco especies forestales amenazadas, sólo dos (2) de ellas, el jigua negro y el guayacán amarillo se encuentran en las zonas de muestreo de este municipio, lo que podría explicarse además de las condiciones biofísicas de los sitios pilotos que se tuvieron en cuenta para el estudio, por la devastación que han sufrido grandes superficies de vocación forestal como consecuencia del desarrollo de actividades de aprovechamiento minero mecanizado. Llama la atención la presencia de la especie jigua negro en todas los municipios y en todos los sitios específicos de muestreo, situación que va permitiendo visualizar la no existencia de amenaza sobre ella; de la misma forma se destaca la no presencia de la especie guayaquil en los municipios de Istmina, Río Quito y Carmen del Darién, hecho que confirma una restricción geográfica de la especie y pone de manifiesto la importancia que recobran los municipios de Juradó y Riosucio cuando se emprendan programas de recuperación de este recurso.

Una alusión especial merecen las especies pino amarillo y guayacán amarillo que hacen presencia en el $60 \%$ de los municipios tenidos en cuenta durante el desarrollo del trabajo. Las situaciones que se destacan son, por una parte, la no

Tabla 1

Presencia de las $\mathbf{5}$ especies forestales amenazadas por municipio

\begin{tabular}{lccccc}
\hline Especie & Istmina & Juradó & Riosucio & Río Quito & Carmen del Darién \\
\hline Jigua Negro & $\mathrm{X}$ & $\mathrm{X}$ & $\mathrm{X}$ & $\mathrm{X}$ & $\mathrm{X}$ \\
Abarco & & $\mathrm{X}$ & $\mathrm{X}$ & $\mathrm{X}$ & $\mathrm{X}$ \\
Pino amarillo & $\mathrm{X}$ & $\mathrm{X}$ & $\mathrm{X}$ & \\
Guayacán Amarillo & $\mathrm{X}$ & $\mathrm{X}$ & $\mathrm{X}$ & \\
Guayaquil & & $\mathrm{X}$ & & & \\
\hline
\end{tabular}




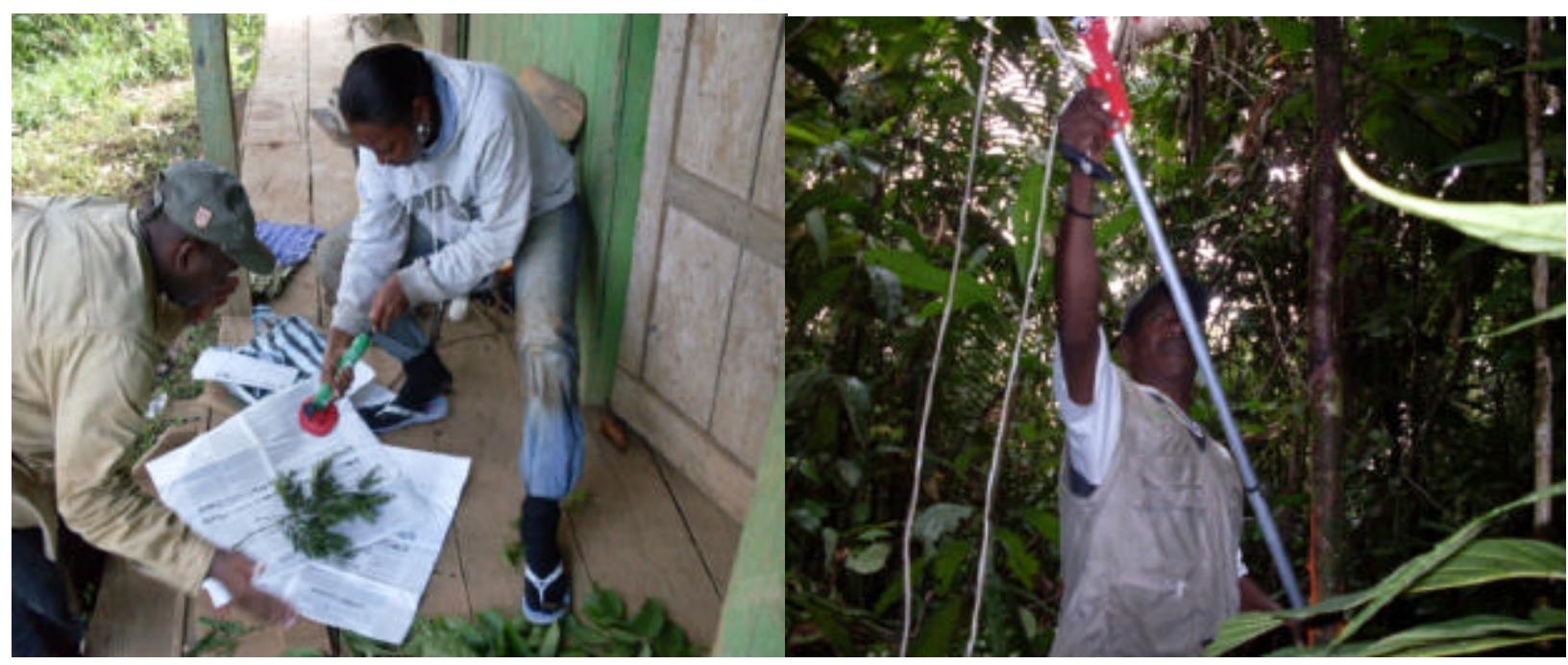

Figura 5. Recolección y preparación de muestras botánicas

presencia de guayacán amarillo en unos municipios que como Riosucio y Carmen del Darién son de altísima vocación forestal y su presencia en el municipio de Istmina, a pesar de las actividades productivas que destruyen la cobertura vegetal de la zona que aún la tiene.

El cuadro también hace evidente la no presencia de la especie abarco en el municipio de Istmina, máxime cuando se registra presencia de ella en todas las otras municipalidades; la reconocida importancia mundial que tiene la especie, es razón suficiente para buscar con seriedad explicaciones a tal situación, lo que supone la revisión de documentos antiguos que den cuenta de la presencia de la especie, el conversatorio con los adultos mayores ubicados en la zona y el análisis de registros de movilización de madera en el lugar, para decidir con fundamento en lo encontrado, la toma de medidas que procuren revertir la situación. La Figura 6 muestra ejemplares de las especies forestales nativas amenazadas abarco, jigua negro, guayaquil, pino amarillo y guayacán encontrados en los sitios de muestreo.

\section{ESTADO DE LAS ESPECIES FORESTALES AMENAZADAS. INFORMACIÓN CUANTITATIVA}

En esta parte del documento se presentan los resultados de la información primaria levantada en los sitios de muestro; en primer lugar se analiza la situación de cada municipio y al final se integra la información para dar cuenta de lo que ocurre con las especies forestales amenazadas en general.

\section{ESTADO DE LAS ESPECIES FORESTALES AMENAZADAS EN CARMEN DEL DARIÉN}

El sitio de muestreo escogido en el municipio de Carmen del Darién fue la comunidad de Chicao; se montaron un total de 62 parcelas y se registró la información que se relaciona a continuación. Se encontraron 220 individuos que representan cinco especies de cinco familias diferentes. La Tabla 2 presenta una relación del número de individuos por especie.

La especie más representativa es el jigua negro de la familia Lauraceae con algo más 264 individuos por hectárea, lo cual da cuenta de su abundancia y de la no existencia de elementos que generen preocupación por su agotamiento es esta zona. Un caso aparte y destacadamente positivo lo constituye la situación del pino amarillo que presentan casi cinco individuos por hectárea en jurisdicción de la comunidad de Chicao, dato que es marcadamente superior al del municipio de Riosucio y cualitativamente muy diciente frente a otros sitios del departamento contemplados en este estudio, en donde ni siquiera se encontró un ejemplar de la especie. La Tabla 3 relaciona la distribución de individuos de las especies forestales amenazadas por estado de desarrollo, los datos hacen evidentes los problemas de regeneración natural que presentan las especies abarco y pino amarillo e inclusive muestran para la especie jigua negro una distribución supremamente irregular en comparación con lo encontrado en los otros sitios objetos del presente estudio.

Esta situación generalizada que muestra más del $80 \%$ de los individuos en estado de fustal, hace notorio el peligro que se cierne sobre las especies forestales amenazadas de no tomarse medidas inmediatas para garantizar su protección.

La Tabla 4 presenta la distribución de individuos de especies forestales amenazadas por clase diamétrica en la comunidad de Chicao, municipio de Carmen del Darién en el departamento del Chocó. Se destaca en esta información el bajo número de individuos de jigua negro que alcanza las clases diamétricas superiores, en contraste con la baja regeneración natural del abarco y la nula regeneración natural del pino amarillo. 


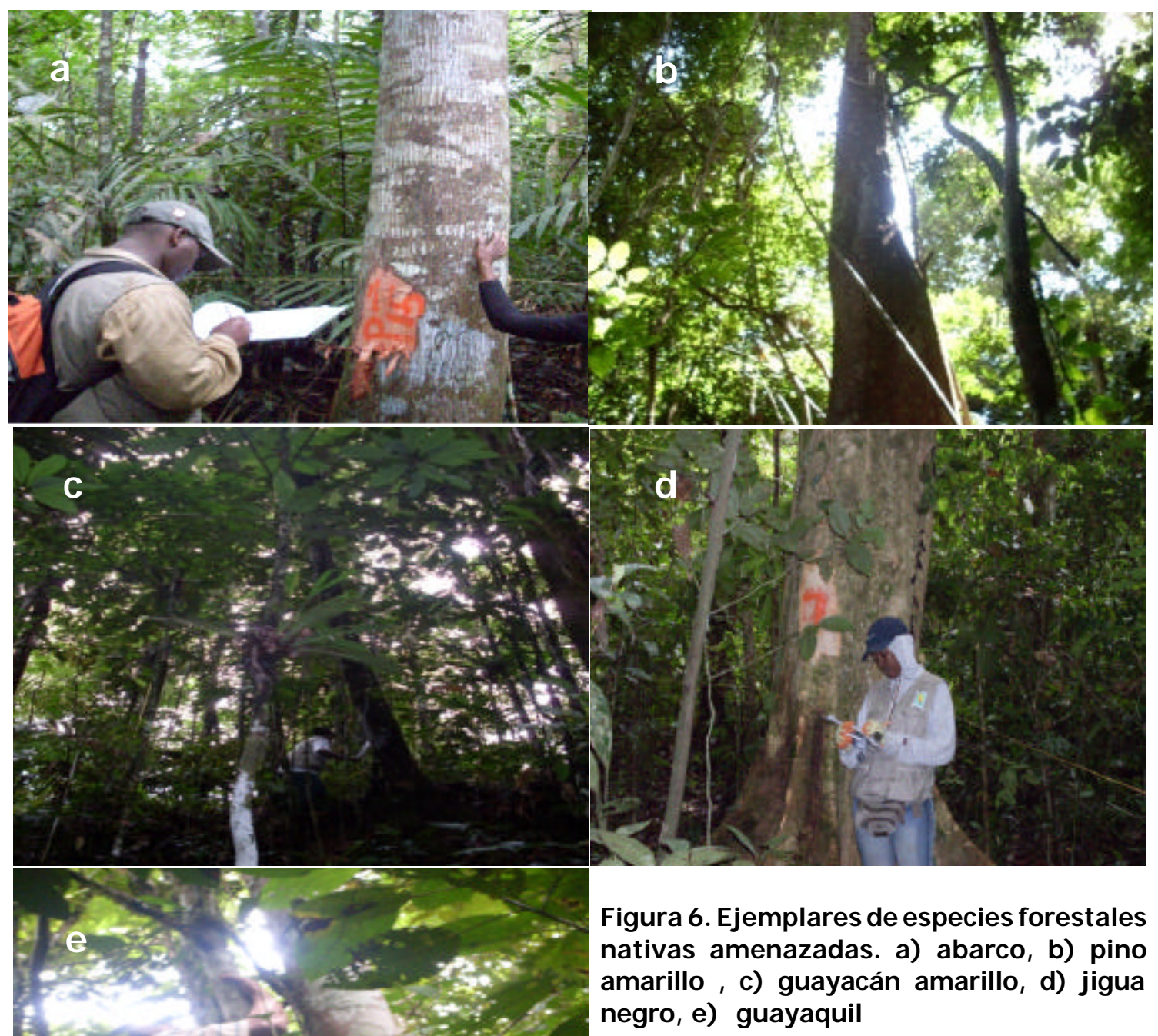

especies. La Tabla 5 presenta información integrada del territorio colectivo del Consejo Comunitario Mayor de Istmina en el departamento del Chocó, correspondiente al levantamiento de 205 parcelas de $10 \mathrm{~m}$ x $10 \mathrm{~m}$ para un total de 2.05 hectáreas, entre Suruco Santa Mónica y Puerto Salazar, vereda Chigorodó.

En el municipio de Istmina la especie más abundante es el jigua negro con 320 individuos por hectárea, lo contrario ocurre con la especie guayacán amarillo, que presenta 19 individuos por hectárea, situación que muestra signos de agotamiento y que evidencia la necesidad de emprender

\section{ESTADO DE LAS ESPECIES FORESTALES AMENAZADAS EN ISTMINA}

En el municipio de Istmina se tuvo acceso a dos sitios de muestreo, uno en el corregimiento de Suruco, vereda Santa Mónica y otro en el corregimiento de Puerto Salazar, vereda de Chigorodó. Sin lugar a dudas en este último sitio se presenta mayor abundancia de individuos e inclusive de procesos de recuperación de esta especie.

En la Tabla 6 se presenta la distribución de individuos por estado de desarrollo; de la información resultante se destaca la amplia presencia del jigua negro con abundante población en todos los estados de crecimiento, muy importante resulta la cantidad de individuos que en estado de brinzal (380), lo que supone un buen comportamiento de la regeneración natural de la especie; los datos en este sentido son claros, no hay 
Tabla 2

Número de individuos por especie forestal amenazada en la comunidad de Chicao, Carmen del Darién, Chocó

\begin{tabular}{lllrr}
\hline Familia & \multicolumn{2}{c}{ Nombre } & Número total de árboles & $\begin{array}{c}\text { Número de árboles } \\
\text { por hectárea }\end{array}$ \\
\cline { 2 - 3 } & científico & vulgar & & \\
\hline Lecythidaceae & Cariniana pyriformis & Abarco & 17 & 27.4 \\
Lauraceae & Ocotea cernua & Jigua negro & 164 & 264.5 \\
Podocarpaceae & Podocarpus sp. & Pino amarillo & 3 & 4.8 \\
Total & & & 184 & \\
\hline
\end{tabular}

Tabla 3

Distribución de individuos de especies forestales amenazadas por estado de desarrollo en Chicao y Carmen del Darién, Chocó

\begin{tabular}{lcrrr}
\hline Especie & Brinzal & Latizal & Fustal & Total \\
\hline Abarco & & 1 & 16 & 17 \\
Jigua negro & 18 & 22 & 124 & 164 \\
Pino amarillo & & & 3 & 3 \\
Total & & & & 184 \\
\hline
\end{tabular}

razones suficientes para mostrar una excesiva preocupación por la posibilidad de agotamiento prematuro de esta especie.

El caso contrario lo constituye la especie forestal guayacán amarillo, donde el número total de individuos es relativamente bajo, con el agravante que los brinzales se encuentran notablemente disminuidos. Aquí resulta evidente la necesidad de proteger los fustales, identificando los árboles semilleros y garantizando su permanencia para poderlos utilizar en futuros programas de manejo y fomento de estas especies.

La Tabla 7 muestra la distribución de los individuos de las especies forestales amenazadas por clase diamétrica, ambas especies, jigua negro y guayacán amarillo muestran una distribución regular al presentar la mayor cantidad de indivi-

Tabla 4

Distribución de individuos de especies forestales amenazadas por clase diamétrica en Chicao, Carmen del Darién, Chocó

\begin{tabular}{lccccccccc}
\hline Especie & \multicolumn{7}{c}{ Clase diamétrica (cm) } & Total \\
\cline { 2 - 7 } & $\mathbf{0 - 1 0}$ & $\mathbf{1 0 - 2 0}$ & $\mathbf{2 0 - 3 0}$ & $\mathbf{3 0 - 4 0}$ & $\mathbf{4 0 - 5 0}$ & $\mathbf{5 0 - 6 0}$ & $\mathbf{7 6 0}$ & \\
\hline Abarco & 1 & 2 & 1 & 2 & 2 & 3 & 6 & 17 \\
Jigua Negro & 40 & 58 & 38 & 16 & 6 & 3 & 3 & 164 \\
Pino Amarillo & & & & 1 & 1 & 1 & & 3 \\
Total & & & & & & & & 184 \\
\hline
\end{tabular}

Tabla 5

Especies forestales amenazadas en el Consejo Comunitario Mayor de I stmina, Chocó

\begin{tabular}{lllcc}
\hline Nombre de la especie & Nombre científico & Familia & $\begin{array}{c}\text { Número de } \\
\text { individuos }\end{array}$ & $\begin{array}{c}\text { Número de individuos } \\
\text { por hectárea }\end{array}$ \\
\hline Jigua negro & Ocotea cernua & Lauraceae & 658 & 320.48 \\
Guayacán amarillo & Tabebuia chrysantha & Bignoniaceae & 39 & 19.02 \\
\hline Total & & & 697 & \\
\hline
\end{tabular}


Tabla 6

Distribución de individuos por estado de desarrollo en el Consejo Comunitario Mayor de Istmina, Chocó

\begin{tabular}{lcccc}
\hline \multirow{2}{*}{ Especie } & \multicolumn{3}{c}{ Número } & \\
\cline { 2 - 4 } & brinzales & latizales & fustales & Total \\
\hline $\begin{array}{l}\text { Jigua negro } \\
\begin{array}{l}\text { Guayacán } \\
\text { amarillo }\end{array}\end{array}$ & 381 & 209 & 68 & 658 \\
\begin{tabular}{l} 
Total \\
\hline
\end{tabular} & 11 & 8 & 20 & 39 \\
\hline
\end{tabular}

importantes; las especies que en el análisis estructural resultan poco importantes, están evidenciando signos de agotamiento. Se evidencia claramente la abundancia de las especies jigua negro y abarco, al tiempo que se destaca la preocupación que genera la presencia de muy pocos individuos de guayacán amarillo, guayaquil, caoba y pino amarillo.

La Tabla 9 presenta la distribución de individuos de especies forestales amenazadas por estado de desarrollo en jurisdicción de la Asociación de Autoridades Indígenas de Juradó, Chocó; se destaca la excelente distribución del jigua negro, especie que definitivamente no se encuentra en peligro de extinción en esta municipalidad y la tan anunciada escasez del guayacán amarillo y el guayaquil. De igual manera se destaca la situación del pino amarillo, especie a la cual le

Tabla 7

Distribución por clase diamétrica de las especies

\begin{tabular}{|c|c|c|c|c|c|c|c|c|}
\hline \multirow[t]{2}{*}{ Especie } & \multicolumn{7}{|c|}{ Clase diamétrica $(\mathrm{cm})$} & \multirow[t]{2}{*}{ Total } \\
\hline & $0-10$ & $10-20$ & $20-30$ & $30-40$ & $40-50$ & $50-60$ & $>60$ & \\
\hline Guayacán amarillo & 28 & 1 & 2 & 2 & 3 & 2 & 2 & 40 \\
\hline Jigua negro & 625 & 17 & 8 & 5 & & 2 & & 657 \\
\hline
\end{tabular}

Tabla 8

Número de individuos por especie en la jurisdicción de J uradó

\begin{tabular}{llrr}
\hline \multicolumn{1}{c}{ Especie } & Nombre científico & Familia & Total \\
\hline Abarco & Cariniana pyriformis & Lecythidaceae & 333 \\
Jigua negro & Ocotea cernua & Lauraceae & 1371 \\
Guayaquil & Centrolobium paraense tul. & Fabaceae & 7 \\
Pino amarillo & Podocarpus sp. & Podocarpaceae & 42 \\
Guayacán amarillo & Tabebuya chrysantha & Bignonaceae & 4 \\
Total & & & 1757 \\
\hline
\end{tabular}

duos en las clases diamétricas inferiores, lo que indica la existencia de una gran cantidad de material vegetal de estas especies que tiene posibilidades de avanzar hacia estado más adultos en los que el volumen de madera sea deseable para emprender labores de aprovechamiento forestal.

\section{ESTADO DE LAS ESPECIES FORESTALES AMENAZADAS EN JURADÓ}

La Tabla 8 muestra el número total de individuos que por especie fueron inventariados durante el desarrollo del trabajo. Las familias Lauraceae y Lecythidaceae con una única especie, tienen tantos individuos que se ubican como las más quedan pocos individuos confinados en espacios geográficos muy específicos, situación que parece ir más allá del municipio de Juradó y representar lo que prácticamente ocurre en todo el departamento del Chocó.

El consolidado de las áreas refleja varios hechos importantes; todas las especies contempladas en el proyecto, se encuentran jurisdicción de los territorios colectivos de autoridades indígenas de Juradó, lo que muestra la enorme importancia de esta área en la perspectiva de instalación de un programa de protección de especies forestales amenazadas en el departamento del Chocó.

En segundo lugar, se confirma la abundancia relativa de la especie jigua negro que presenta en esta área una gran 
Tabla 9 Número de individuos por especies y estado de
desarrollo en la jurisdicción de la Asociación de
Autoridades I ndígenas de J uradó

\begin{tabular}{lcccc}
\hline Nombre común & Brinzal & Latizal & Fustal & Total \\
\hline Abarco & 162 & 59 & 112 & 333 \\
Jigua & 1079 & 172 & 120 & 1371 \\
Guayaquil & 3 & & 4 & 7 \\
Pino amarillo & 28 & 3 & 11 & 42 \\
Guayacán amarillo & & & 4 & 4 \\
Total & & & & 1757 \\
\hline
\end{tabular}

cantidad de individuos, la mayoría de los cuales se encuentra en estado de brinzales, lo que habla por sí solo del potencial que se tiene con el manejo de la regeneración natural de la especie. Adicionalmente, la distribución que presentan los individuos por clases diamétricas presenta una condición ideal, lo que con adecuados programas de manejo podrían garantizar la permanencia de la especie en el tiempo.

Se destaca también la relativa buena presencia del abarco, cuyas existencias alcanzan los 162 individuos en condición de brinzales. Los programas de restricción del aprovechamiento de esta especie parecen haber provocado un buen efecto si se juzga por el número de individuos que tiene en las clases diamétricas superiores.

Hay evidentes signos de preocupación por lo que ocurre con el guayaquil y el guayacán amarillo en Juradó, situación que resulta aun más alarmante si se tiene en cuenta que la especie no tiene presencia o no se encontró en el $50 \%$ de los municipios que fueron objeto del estudio. La presencia de tan solo siete individuos de guayaquil en el área, con más del $50 \%$ en las clases diamétricas superiores, y de cuatro individuos de guayacán amarillo confinados exclusivamente a la categoría de fustales, urge el establecimiento de una veda definitiva del aprovechamiento de estas dos especies.

A pesar del relativo bajo número de individuos de pino amarillo, en total 42, que se presentan en Juradó, la información resulta esperanzadora por cuanto esta especie parece estar casi extinta en el departamento del Chocó. Una mirada integradora de todo el estudio supone la necesidad de emprender de inmediato un programa de manejo del pino amarillo en el Chocó, que tiene como condición necesaria tomar en cuenta la comunidad indígena de este municipio fronterizo.

Finalmente a manera de resumen y tomando en cuenta toda la información cuantitativa de Juradó, se pueden destacar como hechos notables los siguientes:

El alto número de brinzales de la especie jigua negro en comparación con las otras especies y en general el bajo número de plantas en estado de brinzal; en este sentido se pueden tomar como positivo el caso del abarco. Esta situación es preocupante porque no muestra con suficiencia que el sólo establecimiento de vedas pueda ayudar a resolver el problema de la escasez futura de la mayoría de las especies. En igual sentido se puede concluir sobre los latizales.

La situación de especies como el guayacán amarillo y el guayaquil, que se encuentran única o mayoritariamente en condición de fustales, obliga a su inmediata protección, pues las labores de aprovechamiento pueden conducir prontamente al agotamiento definitivo de las mismas, debido a que el bosque se queda sin opciones naturales de renovación. Este hecho denota la necesidad de aunar esfuerzos para realizar trabajos conjuntos que le devuelvan al departamento del Chocó su riqueza forestal perdida, especialmente para el disfrute de nuevas generaciones.

El evidente agotamiento de especies que fueron intencionalmente buscadas en todos los sitios de muestreo, guayaquil, pino amarillo y guayacán amarillo, situación que debe concitar el interés de toda la ciudadanía y hace urgente la intervención de las autoridades ambientales y de las autoridades indígenas y de comunidades negras, para construir modelos particulares de protección que pueden estar por el lado de la constitución de una red de reservas de entidades étnicas para garantizar la defensa de este importante patrimonio natural.

\section{ESTADO DE LAS ESPECIES FORESTALES AMENAZADAS EN RÍO QUITO}

La situación encontrada en el municipio de Río Quito en lo que hace relación exclusiva a las especies forestales nativas amenazadas contempladas en el estudio, es claramente desfavorable a la posibilidad de garantizar la presencia permanente de estas especies en la municipalidad. En la Tabla 10 se consigna información correspondiente a los resultados del trabajo de campo en la comunidad de San Isidro, municipio de Río Quito en el departamento del Chocó. La especie más representativa es el jigua negro que presenta 416 individuos respectivamente, la menos representativa es el guayacán amarillo. El abarco está medianamente representada por 25 individuos.

La Tabla 11 muestra la distribución de individuos de especies forestales amenazadas de San Isidro, municipio de Río Quito, Chocó, conforme su estado de desarrollo; de la información consignada se destaca el alto porcentaje de individuos de abarco en condición de fustal, indicativo de los problemas de regeneración natural que presenta la especie; el alto número de individuos de jigua negro con representación en todos los estados de desarrollo, especialmente en brinzales; el bajo número de individuos de guayacán amarillo, y la inexistencia de guayaquil y pino amarillo, signos inequívocos de agotamiento. 
Bioetnia Volumen 6 № 1 (enero-junio), 2009

Tabla 10

Número de individuos por especie forestal amenazadas en San I sidro, Río Quito, Chocó

\begin{tabular}{lllrr}
\hline Nombre común & Nombre cientifico & Familia & $\begin{array}{r}\text { Número de } \\
\text { individuos }\end{array}$ & $\begin{array}{r}\text { Número de individuos } \\
\text { por hectárea }\end{array}$ \\
\hline Abarco & Cariniana pyriformis & Lecythidaceae & 25 & 12.76 \\
Guayacán amarillo & Tabebuia crysantha & Bignoniaceae & 2 & 1.02 \\
Jigua negro & Ocotea cernua Nees & Lauraceae & 416 & 212.24 \\
Total & & & 443 & \\
\hline
\end{tabular}

Tabla 11

Estado de desarrollo de los individuos de especies forestales amenazadas en territorio del Consejo Comunitario de Río Quito, Chocó

\begin{tabular}{lcccr}
\hline Especie & \multicolumn{3}{c}{ Número } & \\
\cline { 2 - 4 } & brinzales & latizales & fustales & Total \\
\hline Abarco & 1 & 3 & 21 & 25 \\
Jigua negro & 219 & 101 & 96 & 416 \\
Guayacán & & & & \\
amarillo & 1 & 0 & 1 & 2 \\
Guayaquil & 0 & 0 & 0 & 0 \\
Pino amarillo & 0 & 0 & 0 & 0 \\
Total & & & & 443 \\
\hline
\end{tabular}

Tomando en cuenta las cinco (5) especies objeto este de estudio, únicamente el abarco y jigua negro se encuentran con relativa abundancia en el área de jurisdicción del Consejo Comunitario de San Isidro. Sin embargo, para el primero se observan tan sólo 4 individuos en los estados de brinzal y latizal, situación que es preocupante por la importancia comercial que tiene la especie; los individuos en estado fustal probablemente no alcancen el estado de madurez fisiológico que les permitan distribuir de manera natural las semillas, debido a la intervención antrópica.
Para el casojigua negro la situación es bien diferente; esta especie reportó 387 individuos que equivalen a $65.8 \%$ del total inventariado, datos que lo dejan como la especie más abundante; también se identificó una relativa homogeneidad en los informes de los diferentes estados del bosque, lo que hace deducir que la especie presenta un buen comportamiento sucesional, condición que permite adelantar acciones de manejo más flexibles y sostenibles que posibiliten preservar la especie; lo anterior no valida el concepto inicial de categorizar al jigua negro como especie amenazada; sin embargo, se recomienda avanzar en los estudios en otras zonas para validar esta hipótesis.

La Tabla 12 muestra la distribución de los individuos de especies forestales amenazadas por clase diamétrica en la Comunidad de San Isidro, municipio de Río Quito, Chocó.

\section{ESTADO DE LAS ESPECIES FORESTALES AMENAZADAS EN RIOSUCIO}

En general la situación del Medio Truandó en el municipio de Riosucio, con referencia a especies forestales nativas amenazadas es absolutamente preocupante; el panorama muestra especies con claros y evidentes signos de escasez, que encienden las alarmas y obligan a la autoridad ambiental y a las otras instituciones del Sistema Nacional Ambiental a tomar medidas urgentes para frenar la tendencia encontrada. La Tabla 13 muestra el número total de individuos de las

Tabla 12

Distribución de individuos de especies forestales amenazadas por clase diamétrica en San I sidro, Río Quito, Chocó

\begin{tabular}{lccccccccc}
\hline \multirow{2}{*}{ Especie } & \multicolumn{6}{c}{ Clase diamétrica (cm) } & \multirow{2}{*}{ Total } \\
\cline { 2 - 7 } & $\mathbf{0 - 1 0}$ & $\mathbf{1 0 - 2 0}$ & $\mathbf{2 0 - 3 0}$ & $\mathbf{3 0 - 4 0}$ & $\mathbf{4 0 - 5 0}$ & $\mathbf{5 0 - 6 0}$ & $\mathbf{7 6 0}$ & \\
\hline Jigua negro & 334 & 52 & 20 & 5 & 3 & 2 & & 416 \\
Abarco & 4 & 8 & 5 & 2 & 2 & 2 & 2 & 25 \\
Guayacán amarillo & 1 & 1 & & & & & & 2 \\
\hline
\end{tabular}


Estado de conservación de especies forestales amenazadas. W. Klinger-Brahan

Tabla 13

Número de individuos encontrados en Truandó Medio, Riosucio, Chocó

\begin{tabular}{lllrr}
\hline Familia & \multicolumn{2}{c}{ Nombre } & Número total de árboles & $\begin{array}{c}\text { Número de árboles } \\
\text { por hectárea }\end{array}$ \\
\cline { 2 - 3 } & \multicolumn{1}{c}{ científico } & vulgar & & \\
\cline { 1 - 2 } Lecythidaceae & Cariniana pyriformis miers & Abarco & 768 & 339.8 \\
Fabaceae & Centrolobium paraense & Guayaquil & 7 & 3.1 \\
Lauraceae & Ocotea cernua & Jigua negro & 247 & 109.3 \\
Podocarpaceae & Podocarpus sp. & Pino amarillo & 2 & 0.9 \\
Total & & & 1024 & \\
\hline
\end{tabular}

especies forestales amenazadas encontradas en Truandó Medio, municipio de Riosucio.

Los datos muestran que se presentan alarmantes signos de agotamiento en la especie pino amarillo, del cual sólo se encontraron 2 ejemplares en este sitio de muestreo durante el desarrollo del trabajo. Las especies abarco y jigua negro abundan en la zona; el abarco presenta más de 300 individuos por hectárea, mientras que el jigua negro presenta más de 100 , hecho que es claro indicativo de su relativa abundancia.

En la Tabla 14 se presenta información relacionada con el estado de desarrollo de los individuos de especies forestales amenazadas en el Medio Truandó, Riosucio, Chocó; se mira con preocupación la inexistencia de regeneración natural de las especies pino amarillo y guayaquil, lo cual sumado a los criterios anteriores sirve para mostrar la evidente necesidad de proteger estas especies. Entre las especies encontradas la más abundante fue el abarco, con un total de 768 individuos, es relevante tener en cuenta que esta especie tiene buena representación en todos los estratos del bosque inventariado; otra especie de marcada existencias es el jigua negro con 247 individuos.
Tabla 14

\section{Estado de desarrollo de los individuos de especies forestales amenazadas en Truandó Medio y Riosucio, Chocó}

\begin{tabular}{lcccc}
\hline Especie & \multicolumn{3}{c}{$\begin{array}{c}\text { Número de individuo por especies } \\
\text { y por estado }\end{array}$} & \\
\cline { 2 - 4 } & brinzales & latizales & fustales & Total \\
\hline Abarco & 44 & 167 & 574 & 785 \\
Guayaquil & & & 7 & 7 \\
Jigua negro & 40 & 28 & 179 & 247 \\
Pino amarillo & & & 2 & 2 \\
\hline Total & & & & 1024 \\
\hline
\end{tabular}

La Tabla 15 presenta la distribución por clase diamétrica de los individuos de especies forestales amenazadas en el Medio Truandó, municipio de Riosucio, Chocó. Se destaca de esta información la inexistencia de ejemplares de abarco con

Tabla 15

Distribución de individuos de especies forestales amenazadas por clases diamétricas en Truandó Medio, Riosucio, Chocó

\begin{tabular}{|c|c|c|c|c|c|c|c|c|}
\hline \multirow[t]{2}{*}{ Especie } & \multicolumn{7}{|c|}{ Clase diamétrica $(\mathrm{cm})$} & \multirow[t]{2}{*}{ Total } \\
\hline & $0-10$ & $10-20$ & $20-30$ & $30-40$ & $40-50$ & $50-60$ & $>60$ & \\
\hline Abarco & 210 & 324 & 146 & 68 & 16 & 4 & & 768 \\
\hline Carrá & & & & 2 & 2 & & 4 & 8 \\
\hline Choibá & & & 1 & 1 & 2 & 1 & 9 & 14 \\
\hline Guayaquil & & & & 2 & 2 & 1 & 2 & 7 \\
\hline Jigua negro & 68 & 46 & 64 & 45 & 17 & 3 & 4 & 247 \\
\hline Pino amarillo & & & & & 1 & 1 & & 2 \\
\hline Total & & & & & & & & 1046 \\
\hline
\end{tabular}




\section{Bioetnia Volumen 6 № 1 (enero-junio), 2009}

diámetros a la altura del pecho superiores a 60 centímetros, lo que evidencia la intensidad del aprovechamiento a la que esta especie ha sido sometida durante años en la zona, y al mismo tiempo sugiere la necesidad de impedir su aprovechamiento a pesar del alto número de individuos en clases diamétricas inferiores, justamente para posibilitar el paso de los árboles a estados más maduros de desarrollo.

\section{CONCLUSIONES}

Desde el punto de vista conceptual no resulta útil integrar los datos de campo de los municipios estudiados para inferir información sobre las especies forestales amenazadas del Chocó en lo cuantitativo, pues el sistema de muestreo restringido a cinco municipios no escogidos al azar no sólo resulta insuficiente sino también inconveniente para este tipo de propósitos. Sin embargo, desde la perspectiva cualitativa se puede hacer una mirada que conduce a orientaciones generales supremamente útiles para irle avanzando a decisiones y alianzas que conduzcan a defender y mantener la riqueza forestal otrora cierta.

Los resultados muestran con absoluta contundencia que en el departamento del Chocó, al menos en los sitios de muestreo que se utilizaron durante el desarrollo del presente estudio, no existen condiciones para concluir sobre el agotamiento o escasez de la especie forestal Jigua Negro (Ocotea cernea), sus existencias son altas en prácticamente todos las zonas donde se adelantaron labores de campo, por lo que se recomienda llevar a cabo programas de uso sostenible de esta especie, promoviéndola como especie sustituta de otras que por el contrario muestras claros signos de agotamiento. Sin embargo, se advierten problemas de promoción de los individuos hacia etapas de crecimiento más adultas, razón por la que se requiere previamente un estudio orientado al manejo de la regeneración natural de la especie.

Las especies pino amarillo (Podocarpus sp.), guayaquil (Centrolobium paraense), guayacán amarillo (Tabebuia crhysantha), muestran signos inequívocos de agotamiento; los escasos ejemplares que se encuentran en el departamento están en las clases diamétricas superiores y son restringidas a ciertos lugares; tal situación impone la necesidad de una veda inmediata de largo plazo que impida el aprovechamiento de estas especies, pero que al mismo tiempo se acompañe de un programa de repoblamiento que haga más notorio y rápido el enriquecimiento del bosque natural con esta especie.

$\mathrm{El}$ abarco (Cariniana pyriformis), cuyas existencias son algo mayores que las registradas para las especies anteriores y su distribución por categorías diamétricas es más regular, también muestran tendencias hacia la escasez; tal situación sugiere la declaratoria de una veda temporal de mediano plazo sobre su aprovechamiento al tiempo que se promueven programas de manejo orientados a garantizar la permanencia de estas especies.

Las tendencias generales propuestas para el manejo de las especies forestales amenazadas en el departamento del Chocó, pueden presentar cambios cuando se haga una mirada particular por cada uno de los municipios e inclusive sitios de muestreo, en razón a que la tendencia particular de algunas especies puede cambiar por municipio, conforme lo han mostrado los datos que se levantaron en campo y que son los que permiten llegar a las conclusiones de hoy.

Una situación particular se presenta en los municipios de Riosucio y Juradó con el abarco, especie para la cual se ha recomendado en términos generales una veda de mediano plazo y la implementación de programas de manejo; en esta municipalidad los datos reportados para la especie suponen un tratamiento más flexible. Todo aquí parece indicar que en Riosucio y Juradó pueden promoverse programas de uso sostenible que otorguen posibilidades de aprovechamiento de la especie con cupos limitados y con un sistema de monitoreo y vigilancia que impida el abuso de los madereros.

\section{RECOMENDACIONES}

Debe establecerse un efectivo programa de monitoreo y control del aprovechamiento de especies restringidas, pues a pesar del grado de escasez y vulnerabilidad de las especies forestales, en algunos sitios de muestra se observaron actividades de aprovechamiento que van en contravía de las medidas de protección.

En el área objeto de investigación es una zona de alta intervención maderera lo que exige un pronto cuidado a las especies de las cuales se identificaron individuos en estado de fustales, porque se encuentran en situación de vulnerabilidad, debido a que los madereros frecuentan estos espacios y por cuestiones del estudio se hizo una clara demarcación de estos individuos, lo que podría convertirse en un arma de doble filo, porque los visualizan con mucha facilidad.

Es imperante proteger y seleccionar los mejores árboles semilleros, con el propósito de garantizar su permanencia y utilizarlos en futuros programas de manejo y recuperación en las especies con mayor probabilidad, porque estas son muy apetecidas por su dureza y utilidad; esto se supone en el marco de un programa de manejo de las especies en escasez que se recomienda como etapa de trabajo siguiente en los municipios objeto del estudio. En los otros municipios del departamento se deben emprender labores de investigación similares a las realizadas durante el desarrollo de este trabajo.

\section{LITERATURA CITADA}

Andrade, M. G. 2001. Biodiversidad y conservación de la fauna colombiana. En: P. Muñoz (ed.). Memorias. Primer Congreso Colombiano de Zoologia. Instituto de Ciencias Naturales. Bogotá. Universidad Nacional de Colombia; 2001. p. 35-47. 


\section{Estado de conservación de especies forestales amenazadas. W. Klinger-Brahan}

Calderón, E., G. Galeano, N. García (eds.). 2002. Libro Rojo de Plantas Fanerógamas de Colombia. Volumen 1: Chrysobalanaceae, Dichapetalaceae y Lecythidaceae. La serie Libros rojos de especies amenazadas de Colombia. Bogotá, DC: Instituto Alexander von Humboldt, Instituto de Ciencias Naturales-Universidad Nacional de Colombia, Ministerio del Medio Ambiente.

Holdridge, G. L. 1996. Ecología basada en zonas de vidas. San José: Instituto Interamericano de Cooperación para la Agricultura (IICA).

Kubitzki, K. \& S. Renner. 1982. Lauraceae I (Aniba and Aiouea). Flora Neotropica. 31(1): 1-124.

Mayhew, J.E., Newton, A.C. 1998. The silviculture of mahogany. CABY publishg a división of CAB INTERNATIONAL. serie Cabi.wallingfor. Great Britain. 226 p.

Rodríguez M., A. M. Sibile. 1996. Manual de identificación de especies forestales de la subregión andina. Proyecto pd 150/91. Rev. 1 (1). iniaoimt. Universidad de la Molina. Lima. Pg. 489.

Sociedad de Mejoras Públicas de Medellín, 1999. Caracterización de la zona y diseño del centro de rehabilitación de fauna silvestre en el municipio de la pintada, adecuación y dotación del aviario de fauna silvestre en el parque zoológico Santafé y diagnóstico general de la fauna silvestre en Antioquia. Tomo 1. Medellín. Sociedad de Mejoras Públicas de Medellín. 